Sir,

\section{Pars Plana Vitrectomy in Von Hippel Lindau} Disease

A 21-year-old man presented in 1988 complaining of blurred vision in his right eye. His visual acuity was 6/24 right, 6/6 left. He had multiple bilateral retinal angiomata with secondary macular exudation, consistent with Von Hippel Lindau disease. His father had died at the age of 40 years from a cerebral haemorrhage. Systemic screening of the patient revealed no abnormalities.

The angiomata were treated by a combination of transconjunctival cryotherapy and repeated argon laser photocoagulation, with frequent argon laser treatment since then to recurrent and new angiomata (Fig. 1). The right eye received adjunctive proton beam therapy.

In 1994, the left visual acuity reduced to $6 / 36$ due to combined tractional and exudative retinal detachment, refractory to laser treatment. This was managed successfully by pars plana vitrectomy, excision of pre-retinal membranes and endolaser to residual angioma, with resulting resolution of macular exudate. Left visual acuity 6 months postoperatively was 6/6 (Fig. 2).

\section{Discussion}

Von Hippel Lindau disease is an autosomal dominant syndrome of multisystem neoplasia. Retinal angiomatosis is the most common mode of presentation, at a mean age of 25 years. ${ }^{1}$

The management of retinal angiomata depends on their size and position. Larger tumours often need adjunctive cryotherapy or radiotherapy, depending on their location. Vitreoretinal traction occurs as a distinct part of retinal angiomata ${ }^{2}$ and in this case developed after previous successful treatment. Secondary rhegmatogenous retinal detachment occurs and can be managed by conventional surgery. ${ }^{3}$ Tractional retinal detachment in Von Hippel Lindau disease can be managed by pars plana vitrectomy; ${ }^{4}$

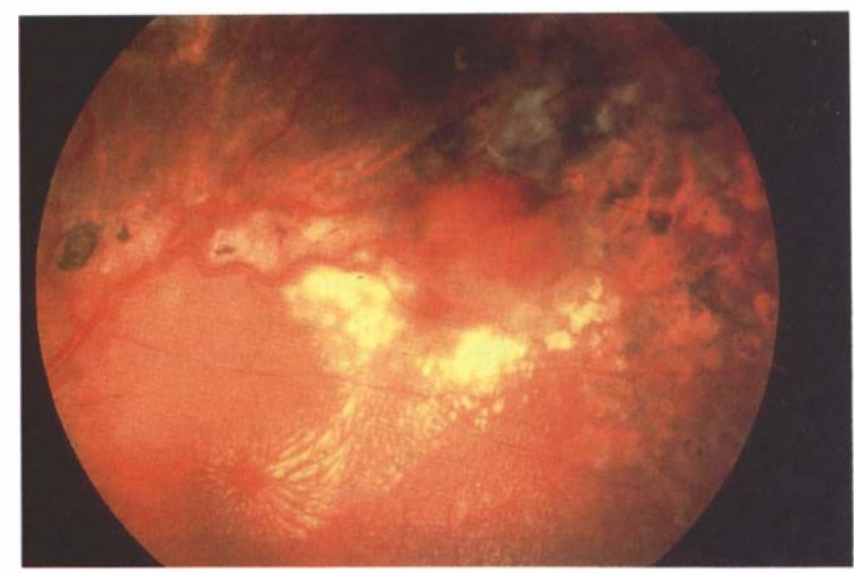

Fig. 1. Left macula 1992. There is recurrent angioma and exudate which responded well to argon laser.

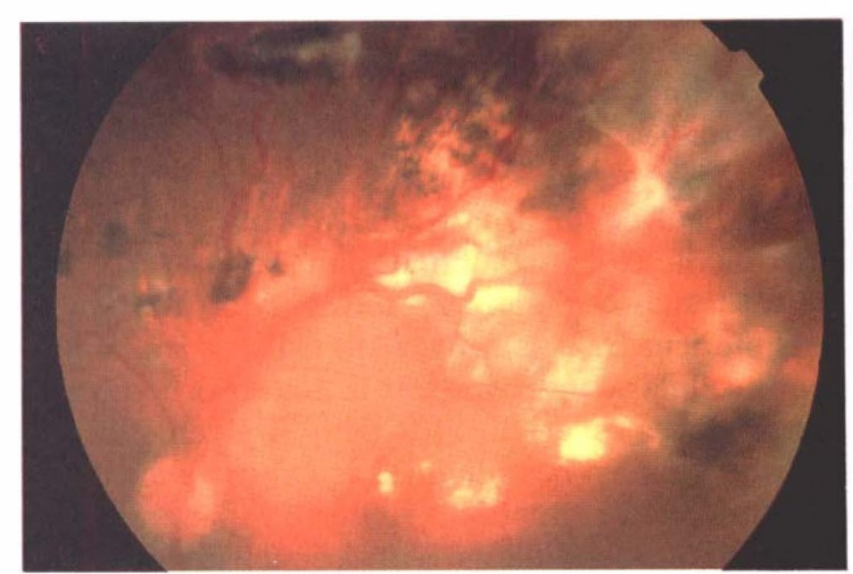

(a)

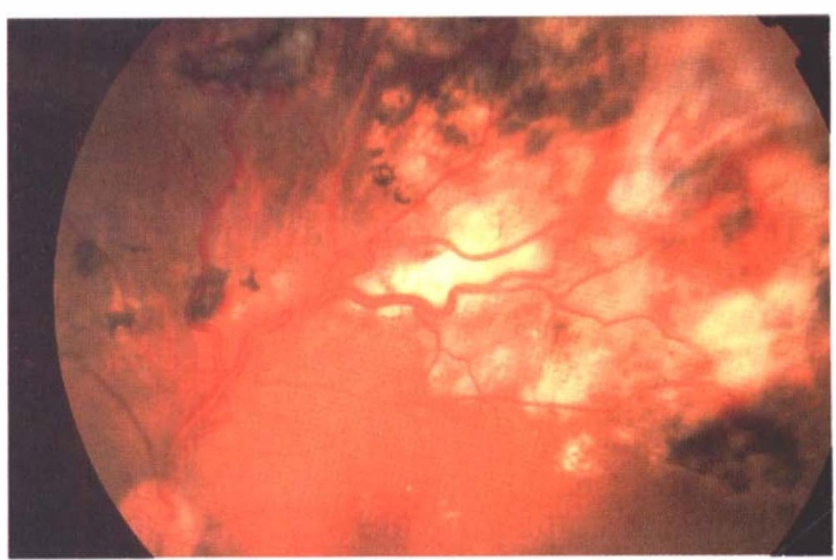

(b)

Fig. 2. (a) Left fundus preoperatively, with tractional and exudative retinal detachment of the macula. Visual acuity was 6/36. (b) Six months after vitrectomy, peeling of preretinal membranes and endolaser there is resolution of macular exudate and continuing treatment of angioma with argon laser. Visual acuity is $6 / 6$.

this is a new and successful intervention in the condition, illustrated by this case.

David A. Mulholland, MRCP, FRCOphth

Patrick B. Johnston, FRCS, FRCOphth

James A. Sharkey, FRCS, FRCOphth

Department of Ophthalmology

Royal Victoria Hospital

Grosvenor Road

Belfast

Northern Ireland

\section{References}

1. Maher ER, Moore AT. Review: Von Hippel Lindau disease. Br J Ophthalmol 1992;76:743-5.

2. Nicholson DH, Green WR, Kenyon KR. Light and electronmicroscopic study of early lesions in angiomatosis retinae. Am J Ophthalmol 1976;82:193.

3. Nicolson DH, Anderson LS, Blodi C. Rhegmatogenous retinal detachment in angiomatosis retinae. Am J Ophthalmol 1986;101:187-9.

4. McDonald HR, Schatz H, Johnson RN, et al. Presenta- 
tion, American Academy of Ophthalmology, 1994. Vitrectomy in eyes with peripheral retinal angiomas associated with traction macular detachment.

Sir,

Regression of Remote Capillary Haemangioma after Local Intralesional Injection of Corticosteroids

Capillary haemangioma is the most common vascular tumour of the ocular adnexa and orbit in infants and children. Spontaneous involution frequently occurs by 4-7 years of age. ${ }^{1}$ Capillary haemangiomas of the eyelids and orbit are best managed by observation, except when vision is threatened by untoward effects of the tumour. When intervention becomes necessary, intralesional corticosteroid injection is preferred.

We report on the beneficial effect of a local intralesional corticosteroid injection on a distant capillary haemangioma in a 16 -week-old infant.

\section{Case Report}

A 3-month-old infant was referred for treatment because of gradually enlarging haemangiomas of the right upper eyelid and the left parotid area which appeared at age 2 months. Initial examination revealed bluish-purple, 'spongy' subcutaneous masses in the right upper eyelid and the left parotid area with telangiectatic vessels. The masses were shown to have irregular margins and rapid uniform enhancement on dynamic computed tomography with iodinated contrast medium. Ocular examination revealed mild proptosis of the right eye with blepharoptosis. The anterior and posterior segments were normal. Cycloplegic refraction was $+2.0-2.5$ $\times 20^{\circ}$ for the right eye and $+0.5-0.25 \times 180^{\circ}$ for the left. Because the lesion in the right upper eyelid was expanding rapidly, with progression of astigmatism to $+3.5-5.25 \times 30^{\circ}$ and the threat of amblyopia, local treatment was recommended. At the age of 16 weeks, a 50:50 mixture of triamcinolone acetonide $(80 \mathrm{mg})$ and betamethasone $(6 \mathrm{mg})$ was injected under general anaesthesia to the right upper eyelid mass. One week later regression of the injected lesion was noted, together with regression of the astigmatism in the right eye to $+3.0-2.0 \times 30^{\circ}$.

Interestingly, concomitant and significant regression of the capillary haemangioma of the left parotid area, which had been excessively enlarged prior to treatment, was also observed.

\section{Discussion}

Patients presenting with orbital capillary haemangiomas can also have coexisting capillary haemangiomas in other parts of the body. To the best of our knowledge, this is the first report of regression of a distant capillary haemangioma after local intralesional injection of corticosteroids. Although regression of the capillary haemangioma lesions may have been due to spontaneous regression, these lesions were characterised by gradual and constant growth prior to the steroid injection, with immediate regression after intralesional injection of corticosteroids. This indicates that regression was due to treatment and not to the natural history of these lesions.

We suggest two explanations for this remote effect. First, the dose of corticosteroids injected intralesionally (triamcinolone acetonide $80 \mathrm{mg}$ and betamethasone $6 \mathrm{mg}$ ) is estimated to be 109-333 times the daily cortisol production of an age-matched infant $(1.8-5.5 \mathrm{mg} /$ day $){ }^{2}$ Second, the amount of corticosteroids that can be absorbed systemically from intralesional injections may be excessive. ${ }^{3}$ This combination of extremely high corticosteroid dose and excessive intravascular absorption may account for not only the response of the distant capillary haemangioma, but also the systemic side effects. Weiss ${ }^{3}$ diagnosed growth retardation in two patients who suffered adrenal suppression after corticosteroid injection into periocular haemangiomas. Therefore, clinicians should take into consideration that intralesional corticosteroid injection into capillary haemangioma may resemble systemic therapy and may cause involution of distant lesions.

David Zadok, MD

Yair Levy, MD

Pinchas Nemet, MD

Department of Ophthalmology

Assaf Harofeh Medical Center

Zerifin 70300

Tel Aviv

Israel

\section{References}

1. Bowers RE, Graham EA, Tomlinson KM. The natural history of the strawberry nevus. Arch Dermatol 1960;82:667-80.

2. Kenny FM, Preeyasombat C, Migeon CJ. Cortisol production rate. II. Normal infants, children and adults. Pediatrics 1966;37:34.

3. Weiss AH. Adrenal suppression after corticosteroid injection of periocular hemangiomas. Am J Ophthalmol 1989;107:518-22.

Sir,

\section{Pseudoretinitis Pigmentosa due to Sub-optimal} Treatment of Neurosyphilis

The treatment of established neurosyphilis requires intensive therapy with frequent, high-dose intravenous penicillin $G$ or high-dose intramuscular repository penicillin $\mathrm{G}$ with probenecid. ${ }^{1}$ However, patients may be treated for neurosyphilis with penicillin in oral or intramuscular form alone. The usual dose prescribed in these situations is inadequate for the complete eradication of the Treponema pallidum organisms from the eye, yet may be 